\title{
液相色谱-串联质谱法检测水产品中残留的 硝基呋喃类药物的代谢物
}

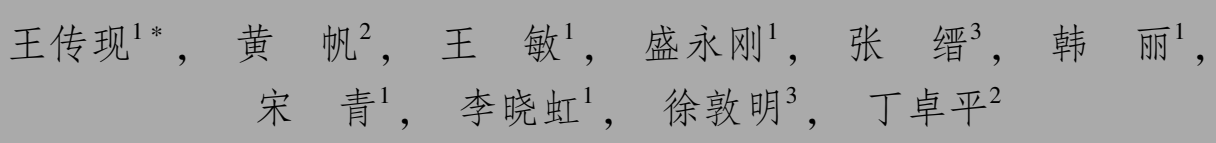

（1. 上海出人境检验检疫局, 上海 200135 ; 2. 上海海洋大学, 上海 201306 ;

3. 厦门出人境检验检疫局, 厦门 361026 )

摘要:建立了液相色谱-串联质谱 (LC-MS/MS) 法用于同时测定水产品中硝基呋喃类药物的代谢物 3-氨基-2-唑烷 基酮（AOZ）、5-甲基吗啉-3-氨基-2-唑烷基酮（AMOZ）、氨基脲（SEM）、1-氨基-2-内酰脲（AHD）和 3,5-二硝基水杨 酸肼 $(\mathrm{DNSH})$ 。样品经盐酸水解、2-硝基苯甲醛衍生、乙酸乙酯提取净化。氮吹至干后, 用 $1 \mathrm{~mL}$ 乙腈- $0.1 \%$ 甲酸水 (20:80, v/v) 定容。经 Aquasil $\mathrm{C}_{18}$ 色谱柱分离, 用液相色谱-三重四极杆串联质谱以多反应监测模式 ( MRM) 进行 检测分析,内标法定量。结果表明,该方法的线性范围为 $0.5 \sim 10 \mu \mathrm{g} / \mathrm{kg}, 5$ 种代谢物的线性相关系数均不小于 0.9976 , 定量限为 $0.5 \mu \mathrm{g} / \mathrm{kg}$ 。在 $0.5 、 1.0 、 2.0$ 和 $4.0 \mu \mathrm{g} / \mathrm{kg}$ 的添加水平下, 加标回收率为 $81.3 \% \sim 100.5 \%$, RSD 为 3. $4 \% \sim 10.0 \%$ 。本法可作为水产品中 5 种硝基呋喃类药物的代谢物残留量同时分析的有效手段。 关键词: 液相色谱-串联质谱法; 硝基呋喃; 代谢物; 水产品

中图分类号: O658 文献标识码:A 文章编号:1000-8713(2013)03-0206-05

\section{Determination of metabolite residues of nitrofuran antibiotics in aquatic products by liquid chromatography-tandem mass spectrometry}

WANG Chuanxian $^{1 *}$, HUANG Fan ${ }^{2}$, WANG Min ${ }^{1}$, SHENG Yonggang ${ }^{1}$, ZHANG Jin ${ }^{3}$, HAN Li ${ }^{1}$, SONG Qing ${ }^{1}$, LI Xiaohong ${ }^{1}$, XU Dunming ${ }^{3}$, DING Zhuoping ${ }^{2}$ (1. Shanghai Exit-Entry Inspection and Quarantine Bureau, Shanghai 200135, China;

2. Shanghai Ocean University, Shanghai 201306, China;

3. Xiamen Exit-Entry Inspection and Quarantine Bureau, Xiamen 361026, China)

Abstract: A method was developed for simultaneous qualitative and quantitative analysis of five metabolites of nitrofuran antibiotics, including 3-amino-2-oxazolidinone ( AOZ), 5-morpholino-methyl-3-amino-2-oxazolidinone ( AMOZ ), semicarbazide ( SEM ), 1-aminohydantoin (AHD) and 3,5-dinitrosalicylic acid hydrazide (DNSH) in aquatic products by liquid chromatography-tandem mass spectrometry ( LC-MS/MS). The samples were hydrolyzed with $\mathrm{HCl}$, and derivatized with 2-nitrobenzaldehyde at $37{ }^{\circ} \mathrm{C}$ for 16 hours. The derivative solutions were adjusted to $\mathrm{pH} 7.0-7.5$, and the analytes were extracted by ethyl acetate. The separation was based on Thermo Aquasil $\mathrm{C}_{18}$ column $(150 \mathrm{~mm} \times 4.6 \mathrm{~mm}, 3.0 \mu \mathrm{m})$. The analytes were detected by tandem mass spectrometry with electrospray ionization source with multiple reaction monitoring ( MRM) mode. The developed method showed good linear correlation between the peak area ratios of the analyte and the internal standard and the concentration of the analyte with the correlation coefficients all above 0.99 over the dynamic range of $0.5-10 \mu \mathrm{g} / \mathrm{kg}$. The limits of quantitation (LOQs) of AOZ, AMOZ, SEM, AHD and DNSH were $0.5 \mu \mathrm{g} / \mathrm{kg}$. The

* 通讯联系人. Tel: ( 021 )38620542,E-mail : chuanxian. wang@ 163.com.

基金项目: 上海市科委重点科技攻关项目(10391901800); 上海市科委长三角联合攻关项目(11495810200); 上海市科委工程中心建 设项目 $(11 \mathrm{DZ} 2280300)$.

收稿日期: 2012-11-20 
average recoveries of all the compounds at four spiked levels of $0.5,1.0,2.0$ and $4.0 \mu \mathrm{g} / \mathrm{kg}$ ranged from $81.3 \%$ to $100.5 \%$ with the RSDs between $3.4 \%$ and $10.0 \% \quad(n=6)$. The method is proved to be fast and effective for simultaneous qualitative and quantitative analysis of the metabolites of the nitrofuran antibiotics in aquatic products.

Key words: liquid chromatography-tandem mass spectrometry ( LC-MS/MS); nitrofuran; metabolites; aquatic products

硝基呋喃类抗生素是一类人工合成的具有 5硝基结构的广谱抗菌药物, 是治疗畜禽胃肠道疾病 的药物或者饲料添加剂。硝基呋喃类药物具有慢性 毒性, 可引起消化道反应, 如恶心、呕吐、厌食、腹泻 等,还可引起溶血性贫血和黄疸。长时间或大剂量 使用硝基呋喃类药物均能对动物体产生毒性作用, 还具有致癌、致畸、致突变作用 ${ }^{[1]}$ 。硝基呋喃类药 物在动物体内的半衰期短, 代谢速度快。但其代谢 产物能够与组织蛋白质紧密结合, 以结合态形式在 体内残留较长时间, 甚至经过蒸煮、烘烤、磨碎和微 波加热也无法有效降解, 且毒性更强。因此, 检测硝 基呋喃类药物的代谢物更能起到监控作用 ${ }^{[2-4]}$ 。

目前国内外关于硝基呋喃类药物及其代谢产物 的检测方法主要有分光光度法 ${ }^{[5]}$ 、高效液相色谱 法 ${ }^{[6-9]}$ 、液相色谱-质谱联用分析法 ${ }^{[10-12]}$ 和免疫分 析法 ${ }^{[13-15]}$ 等。当前国际上普遍采用液相色谱-串联 质谱法对其进行监测和确证。Verdon 等 ${ }^{[16]}$ 建立了 同时检测 5 种硝基呋喃类药物代谢物的多残留的液 相色谱-质谱法。Kaufmann 等 ${ }^{[17]}$ 建立了检测包括 硝呋索尔在内的 5 种硝基呋喃类药物的液相色谱-

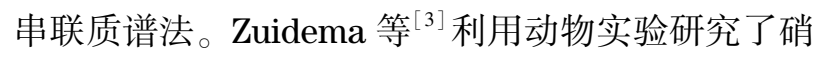
呋索尔在肉鸡中的代谢及降解, 并建立了液相色谱串联质谱法对肉鸡的不同部位残留的硝呋索尔及其 代谢产物 3,5-二硝基水杨酸肼( DNSH) 进行检测。

欧盟于 1995 年发布指令不再允许硝基呋喃类 药物使用在食用动物身上。新发现的呋喃类药物硝 呋索尔 (nifursol) 也被欧盟 1756/2002/EC 禁用。 我国无论在科研方面还是在标准制定方面,对硝呋 索尔的检测研究还比较欠缺, 急需建立相应的检测 方法, 以满足进出口贸易的需要。国外对硝呋索尔 的研究大多基于禽兽肉中的残留检测, 对于水产品 中的检测相对较少。故本研究建立了液相色谱-串 联质谱法检测鱼、虾等基质中 5 种硝基呋喃类药物 的代谢物残留量的方法。

\section{1 实验部分}

\section{1 仪器、试剂与材料}

LC-20ADXR 高效液相色谱仪( 日本 Shimadzu
公司); API 4000 四极杆串联质谱仪 (美国 AB 公 司); Thermo Aquasil $\mathrm{C}_{18}$ 色谱柱 ( $150 \mathrm{~mm} \times 4.6$ $\mathrm{mm}, 3.0 \mu \mathrm{m}$, 美国 Thermo 公司); 德国 IKA HS260 型数显往复式摇床; Milli-Q 高纯水发生器 (美国 Millipore 公司)。

硝基呋喃类药物的代谢物标准及内标物质: DNSH ( 俄罗斯 Vitas-M 公司)；1-氨基-2-内酰腿 (AHD)、氨基艮 (SEM)（德国 Dr. Ehrenstorfer 公 司 ）３-氨基-2-唑烷基酮（AOZ）、5-甲基吗啉-3-氨 基-2-唑烷基酮 (AMOZ)、硝呋奇特和氛、 ${ }^{13} \mathrm{C} 、{ }^{15} \mathrm{~N}$ 标 记的 AOZ-D $、$ AMOZ- $D_{5} 、{ }^{13} \mathrm{C}_{3}-\mathrm{AHD} 、 \mathrm{SEM}-{ }^{13} \mathrm{C}^{15} \mathrm{~N}_{2}$ (德国 Witega 公司)。纯度均大于 $99 \%$ 。水杨酰肼 $(\mathrm{SAH})$ 由硝呋奇特水解获得。

甲醇、乙酸乙酯、正己烷 (HPLC 级, J. T. Baker 公司); 乙腈 (HPLC 级, Promptar 公司); 甲酸 (美国化学学会标准级,J. T. Baker 公司); 二甲亚 砜 (HPLC 级, Sigma 公司); 2-硝基苯甲醛(纯度 > 98\%, Sigma 公司); 盐酸(分析纯,昆山金城试剂有 限公司); 磷酸氢二钠 (分析纯, 上海新华化工厂); 氢氧化钠 (分析纯, 中国医药上海化学试剂公司)。 盐酸溶液, $1.0 \mathrm{~mol} / \mathrm{L}$; 磷酸氢二钠溶液, $0.1 \mathrm{~mol} / \mathrm{L}$; 氢氧化钠溶液, $1.0 \mathrm{~mol} / \mathrm{L}$ 。衍生剂: 称取 $75.5 \mathrm{mg}$ 2-硝基苯甲醛并溶解于 $10 \mathrm{~mL}$ 二甲亚砜中, 现配现 用。实验用水为超纯水 (Millipore 系统生产)。

样品: 鱼、虾和蟹均购于本地市场, 样品使用前 均去壳、去骨。

\section{2 样品前处理}

\subsection{1 水解及衍生化}

称取 $1.0 \mathrm{~g}$ 均质试样于 $50 \mathrm{~mL}$ 塑料离心管中, 依次加人 $10 \mathrm{~mL}$ 水、 $0.5 \mathrm{~mL}$ 盐酸溶液和 $500 \mu \mathrm{L} 2$ 硝基苯甲醛溶液, 混匀后置于 $37{ }^{\circ} \mathrm{C}$ 恒温振荡器中保 持 $16 \mathrm{~h}$ 。

\subsection{2 净化}

将经水解、衍生后的样品溶液取出后, 加人 0.1 $\mathrm{mol} / \mathrm{L}$ 磷酸氢二钠溶液 $5 \mathrm{~mL}$, 用 $1.0 \mathrm{~mol} / \mathrm{L}$ 氢氧化 钠溶液调节 $\mathrm{pH} 7.0 \sim 7.5$ 。在该中性溶液中加人 $2.0 \mathrm{~g}$ 中性氧化铝, 摇匀 $1 \mathrm{~min}$, 于 $10{ }^{\circ} \mathrm{C}$ 下以 4000 $\mathrm{r} / \mathrm{min}$ 离心 $3 \mathrm{~min}$ 。上层清液转移至装有 $16 \mathrm{~mL}$ 乙 
酸乙酯的塑料离心管中, 振荡提取。再于 $10{ }^{\circ} \mathrm{C}$ 下以 $4000 \mathrm{r} / \mathrm{min}$ 离心 $3 \mathrm{~min}$, 使其分层。将上清液转移 至 $20 \mathrm{~mL}$ 氮吹管中, 再加人 $10 \mathrm{~mL}$ 乙酸乙酯重复振 荡提取。经上述步骤后, 合并提取液, 提取液在 40 ${ }^{\circ} \mathrm{C}$ 下用氮气吹干。吹干后, 在氮吹管中加人 $1 \mathrm{~mL}$ 乙腈- $0.1 \%$ (如无特殊说明均为体积分数) 甲酸溶液 (20: 80 , 体积比)。再加人 $2 \mathrm{~mL}$ 乙腈饱和的正己烷 去脂。下层溶液转移至 $2 \mathrm{~mL}$ 离心管中, 以 16000 $\mathrm{r} / \mathrm{min}$ 离心 $5 \mathrm{~min}$, 取上清液过 $0.2 \mu \mathrm{m}$ 滤膜后转移 至进样小瓶中待测。

\section{3 色谱/质谱条件}

Thermo Aquasil $\mathrm{C}_{18}$ 色谱柱 $(150 \mathrm{~mm} \times 4.6$ $\mathrm{mm}, 3.0 \mu \mathrm{m})$ 。流动相 $\mathrm{A}$ 为含 $0.1 \%$ 甲酸的乙腈, $\mathrm{B}$ 为含 $0.1 \%$ 甲酸的 $5 \mathrm{mmol} / \mathrm{L}$ 乙酸铵溶液。梯度 洗脱程序: $0 \sim 4 \mathrm{~min}, 10 \% \mathrm{~A} \sim 99 \% \mathrm{~A}$; $4 \sim 8 \mathrm{~min}$, $99 \% \mathrm{~A} ; 8 \sim 8.5 \mathrm{~min}, 99 \% \mathrm{~A} \sim 10 \% \mathrm{~A}_{\circ}$ 流速: 0.6 $\mathrm{mL} / \mathrm{min}_{\circ}$ 进样量: $20 \mu \mathrm{L}$ 。柱温: 常温

采用电喷雾离子源; 喷雾电压( IS) : $5500 \mathrm{~V}$; 雾 化气压力 $(\mathrm{GS} 1)$ : $70 \mathrm{psi}(482.6 \mathrm{kPa})$; 气帘气压力 $(\mathrm{CUR}): 35 \mathrm{psi}(241.3 \mathrm{kPa})$; 辅助气压力 $(\mathrm{GS} 2)$ : $60 \mathrm{psi}(413.7 \mathrm{kPa})$; 离子源温度 $(\mathrm{TEM}): 550{ }^{\circ} \mathrm{C}$ 。 定性离子对和定量离子对等其他参数见表 1 。

\section{2 结果与讨论}

\section{1 前处理条件的优化}

硝基呋喃类药物的代谢物以蛋白质结合物的形 态存在于样品组织中, 并在适当的酸性条件下释放 出来。这些代谢物的相对分子质量在 $75 \sim 242$ 之 间,分子太小, 不易产生具有典型特征的离子碎片, 检测灵敏度很低。因此,需要对代谢物进行衍生化。 目前采用最多的衍生化试剂是 2-硝基苯甲醛。本 研究选用 2-硝基苯甲醛作为衍生化试剂, 对水解游 离出来的硝基呋喃类药物的代谢物的自由氨基进行 衍生化,形成的衍生化产物具有较好的质谱特性。 参照文献 ${ }^{[18]}$ 方法, 对食品中的硝基呋喃代谢物进行 了同步水解和衍生化。配制 2-硝基苯甲醛的溶剂 一般为二甲亚砜和甲醇, 本实验对两种溶剂进行比 较。结果发现用甲醇作溶剂, 其衍生物质谱信号强 度较二甲亚砜作溶剂时低, 且背景干扰相对大。因 此, 选定用二甲亚砜配制 2-硝基苯甲醛。

本文考察了两种衍生方式。一是样品直接用 $\mathrm{HCl}$ 溶液均质后, 加衍生剂在 $37{ }^{\circ} \mathrm{C}$ 条件下水解衍 生; 二是样品中加人 $2 \mathrm{~g}$ 中性氧化铝后再加 $\mathrm{HCl}$ 溶 液均质, 加衍生剂在 $37{ }^{\circ} \mathrm{C}$ 条件下水解衍生。实验结 果表明, 衍生时加人中性氧化铝, 对衍生有一定干
扰, 使衍生不完全, 结果的重复性差; 并且对水解时 要求的酸性环境有影响。因此,本实验采用第一种 衍生方式。加人中性氧化铝能吸附样品中的脂肪, 有利于净化步骤, 使提取更完全。故选择在调节溶 液 $\mathrm{pH}$ 值至中性后加人中性氧化铝, 所得的水溶液 澄清, 去脂效果明显。

液液萃取适用于大多数的复合型基质, 其操作 较为简单且成本低廉 ${ }^{[19,20]}$ 。采用乙酸乙酯液液萃 取法进行提取净化, 为了防止乳化,在进行提取之前 离心除去固体样品。对于虾仁、鱼等含脂量高的样 品,也可通过提高离心转速, 降低乳化的现象, 提高 回收率 ${ }^{[21]}$ 。由于乙酸乙酯对水中脂类物质也有一 定的提取作用,在浓缩定容后采用正己烷去脂及高 速离心可有效去除杂质。

\section{2 质谱条件的优化}

采用 API 4000 型液质联用仪的多通道扫描技 术,可以在不同的通道对各组分进行监控,因而不需 要通过调节液相色谱条件将各组分分离开。按质谱 测定的一般要求,先对各化合物进行全扫描,随后对 确定的每个化合物的扫描离子, 采用多反应监测模 式以提高其检测灵敏度。AOZ、AMOZ、SEM、AHD 含有氨基, 在电离情况下容易获取正电荷, 因此选择 正离子模式进行扫描。而 DNSH 除了具有氨基外, 还有羟基, 在电离情况下有获得正、负电荷的可能。 根据实验比对,在负离子模式下, DNSH 具有较高的 信号响应值及较低的噪声。因此我们对 DNSH 选 择负离子扫描模式。由于以负离子模式检测的 DN$\mathrm{SH}$ 和以正离子模式检测的 SEM 、AHD 出峰时间接 近,不能采用正、负离子模式切换的方式进行分离, 故将待测样品以正、负离子模式分别检测。

将 $10 \mathrm{mg} / \mathrm{L}$ 的标准溶液进行衍生化处理, 利用 针洜分别以流动注射的方式在电喷雾模式下进行母 离子和子离子扫描, 并优化去簇电压、碰撞气电压等 参数条件, 以达到最佳灵敏度。优化后的质谱条件 如表 1 所示。

\section{3 液相色谱方法的优化}

对硝基呋喃类药物的代谢物采用 Thermo Aquasil $\mathrm{C}_{18}$ 色谱柱进行分离, 比较了两种不同流动 相甲醇-乙酸铵、乙腈-乙酸铵对待测物离子化程度 的影响。

实验表明, 乙腈 $-5 \mathrm{mmol} / \mathrm{L}$ 乙酸铵作为流动相 时, 5 种代谢物的衍生物响应强度较稳定。考虑到 提高待测物离子化效率, 在流动相中加人了 $0.1 \%$ 甲酸。 $\mathrm{AOZ} 、 \mathrm{AHD} 、 \mathrm{AMOZ} 、 \mathrm{SEM} 、 \mathrm{DNSH}$ 的多反应监 测色谱图如图 1 所示。 
表 1 硝基呋喃类药物的代谢物的多反应监测离子对 以及质谱相关参数

Table 1 Mass spectrometry parameters and multiple reaction monitoring ion pairs of nitrofuran metabolites

\begin{tabular}{llrrr}
\hline \multicolumn{1}{c}{ Compound } & $\begin{array}{l}\text { Qualitative ion } \\
\text { pairs }(m / z)\end{array}$ & $\begin{array}{r}\mathrm{DP} / \\
\mathrm{eV}\end{array}$ & $\begin{array}{r}\mathrm{CE} / \\
\mathrm{eV}\end{array}$ & $\begin{array}{c}\mathrm{CXP} / \\
\mathrm{eV}\end{array}$ \\
\hline $\mathrm{AHD}$ & $249.0>134.1^{*}$ & 69 & 19 & 13 \\
& $249.0>104.1$ & 70 & 32 & 13 \\
${ }^{13} \mathrm{C}_{3}$-AHD & $252.2>134.2$ & 66 & 18 & 13 \\
$\mathrm{AOZ}$ & $236.2>134.1^{*}$ & 70 & 17 & 13 \\
& $236.2>104.1$ & 70 & 15 & 13 \\
$\mathrm{D}_{4}$-AOZ & $240.2>134.2$ & 66 & 18 & 13 \\
$\mathrm{SEM}$ & $209.3>192.1^{*}$ & 60 & 17 & 13 \\
& $209.3>166.2$ & 60 & 15 & 13 \\
${ }^{13} \mathrm{C}^{15} \mathrm{~N}_{2}$-SEM & $212.2>168.2$ & 60 & 15 & 13 \\
$\mathrm{AMOZ}$ & $335.3>291.2 *$ & 60 & 19 & 13 \\
& $335.3>262.2$ & 60 & 25 & 13 \\
$\mathrm{D}_{5}$-AMOZ & $340.3>296.4$ & 66 & 18 & 13 \\
$\mathrm{DNSH}^{*}$ & $374.6>182.0 *$ & -114 & -30 & -13 \\
& $374.6>226.0$ & -114 & -30 & -15 \\
$\mathrm{SAH}$ & $286.0>121.1$ & 45 & 15 & 13 \\
\hline
\end{tabular}

* Quantitative ion pairs. DP: declustering potential; $\mathrm{CE}$ : collision energy; CXP: cell exit potential. AHD: 1-aminohydantoin; AOZ: 3-amino-2-oxazolidinone; SEM: semicarbazide; AMOZ: 5-morpholino-methyl-3-amino-2-oxazolidinone; DNSH: 3,5-dinitrosalicylic acid hydrazide; SAH: salicylhydrazide.

\section{4 基质效应及内标物的选择}

采用液相色谱-串联质谱法进行食品中药物残 留检测时,由于基质效应的影响,导致目标化合物发 生离子增强或抑制作用。通过添加内标物和用空白 样品提取液稀释配制标准工作溶液两种方式相结 合,可以减弱离子化时的基质效应,减少定量结果的 偏差。

内标的使用减小了繁琐的前处理过程对最终定 量结果准确度的影响。分别采用 $\mathrm{AMOZ} 、 \mathrm{AHD}$ 、 $\mathrm{SEM} 、 \mathrm{AOZ}$ 这 4 种分析物各自的同位素标记物 $\left(\mathrm{D}_{5}\right.$ $\left.\mathrm{AMOZ} 、{ }^{13} \mathrm{C}_{3}-\mathrm{AHD} 、{ }^{13} \mathrm{C}^{15} \mathrm{~N}_{2}-\mathrm{SEM} 、 \mathrm{D}_{4}-\mathrm{AOZ}\right)$ 作为内标 定量,减小了外标法对回收率的影响,使定量更加准 确。在对 DNSH 进行测定时发现, 信号强度变化 大,分析的准确度低,与另外 4 种硝基呋喃类代谢物 相比,DNSH 的离子信号强度更低,同时缺少同位素 内标。因此, 在本实验中, DNSH 的内标选择用 DN$\mathrm{SH}$ 的类似物 $\mathrm{SAH}$ (水杨酰肼) 代替, $\mathrm{SAH}$ 是由另一 种硝基呋喃类药物硝呋奇特经过水解获得, 由所得 离子信号能得到满意的分析结果。

\section{5 线性范围及检出限}

在优化实验条件下, 以罗非鱼的空白基质溶液 配制 $0.5 、 1 、 2 、 5 、 10 \mu \mathrm{g} / \mathrm{L}$ 的系列混合标准溶液进行 测定, 以内标法定量 (内标 $50 \mu \mathrm{g} / \mathrm{L}$ ), 以分析物与内

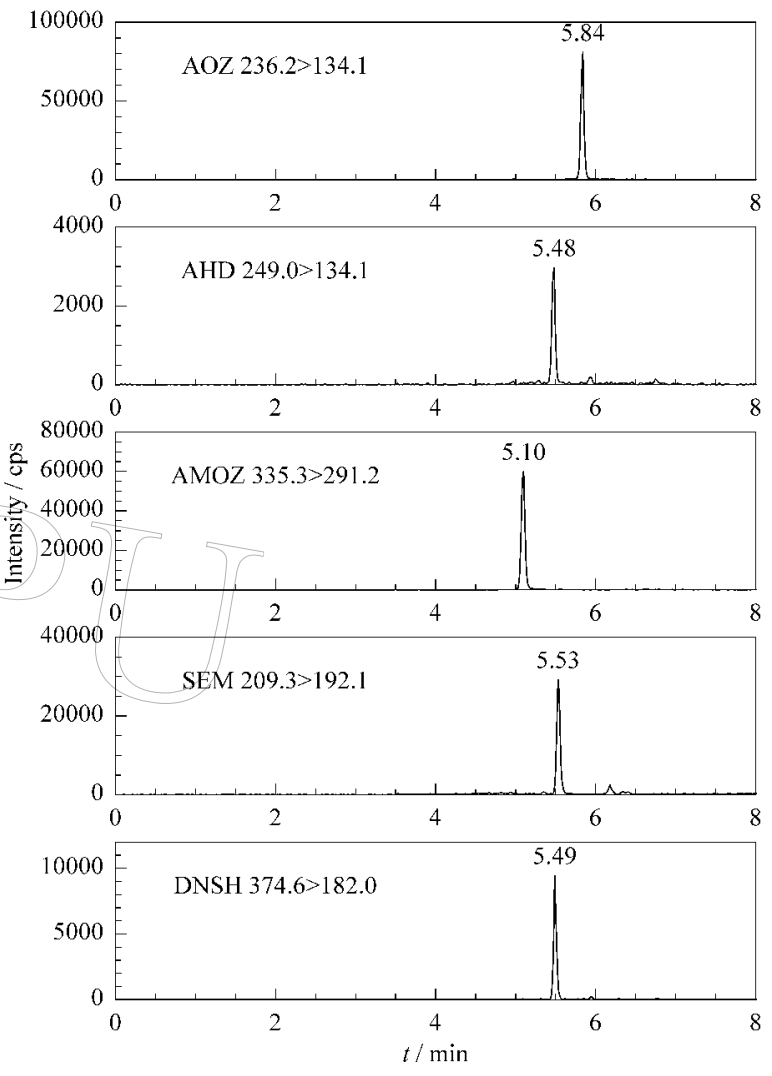

图 1 罗非鱼基质中添加水平为 $1.0 \mu \mathrm{g} / \mathrm{kg} \mathrm{AOZ、AHD、}$ AMOZ、SEM、DNSH 时的多反应监测色谱图

Fig. 1 Multiple reaction monitoring (MRM) chromatograms of AOZ 、AHD 、AMOZ、 SEM,DNSH at the spiked level of 1.0 $\mu \mathrm{g} / \mathrm{kg}$ in Tilapia

标物峰面积比值 $(y)$ 对分析物浓度 $(x)$ 进行线性回 归,结果如表 2 所示。5 种硝基呋喃类药物的代谢 物在 $0.5 \sim 10 \mu \mathrm{g} / \mathrm{L}$ 范围内线性良好, 相关系数 $\left(r^{2}\right)$ 均不小于 0.9976 。以 3 倍信噪比 $(S / N)$ 确定 5 种 硝基呋喃类药物的代谢物的检出限 (LOD) 为 0.2 $\mu \mathrm{g} / \mathrm{kg}$, 以 10 倍信噪比确定其定量限 (LOQ) 为 0.5 $\mu \mathrm{g} / \mathrm{kg}$ 。

表 25 种硝基呋喃类药物的代谢物的线性关系和相关系数 $\left(\boldsymbol{r}^{2}\right)$

Table 2 Linear relationships and correlation coefficients $\left(r^{2}\right)$ of the five nitrofuran metabolites

\begin{tabular}{ccc}
\hline Compound & Regression equation & $r^{2}$ \\
\hline SEM & $y=0.740 x+0.0598$ & 0.9981 \\
AOZ & $y=0.239 x+0.0009$ & 0.9990 \\
AHD & $y=0.364 x-0.0126$ & 0.9982 \\
AMOZ & $y=0.396 x+0.0038$ & 0.9976 \\
DNSH & $y=0.164 x-0.0066$ & 0.9979 \\
\hline
\end{tabular}

$x$ : mass concentration of the analyte, $\mu \mathrm{g} / \mathrm{L} ; y$ : peak area ratio of the analyte and the internal standard.

\section{6 添加回收试验}

在罗非鱼的空白基质中分别添加 5 种硝基呋喃 类药物的代谢物混合标准溶液及内标混合标准溶 液,按前述方法进行添加回收试验和精密度试验, 计 
算平均回收率和相对标准偏差 (RSD, $n=6$ ), 结果 见表 3 。在 $0.5 、 1.0 、 2.0$ 和 $4.0 \mu \mathrm{g} / \mathrm{kg}$ 的添加水平 下, 5 种化合物的平均回收率在 $81.3 \% \sim 100.5 \%$ 之
间, RSD 为 $3.4 \% \sim 10.0 \%$ 。当目标分析物检出值超 出方法的线性范围, 则稀释一定倍数, 使之在线性范 围内。

表 3 罗非鱼中硝基呋喃类药物的代谢物添加回收率范围 $(\boldsymbol{n}=\mathbf{6})$

Table 3 Recovery ranges of nitrofuran metabolites in Tilapia $(n=6)$

\begin{tabular}{|c|c|c|c|c|c|c|c|c|}
\hline \multirow{3}{*}{ Compound } & \multicolumn{8}{|c|}{ Spiked level/ $(\mu \mathrm{g} / \mathrm{kg})$} \\
\hline & \multicolumn{2}{|c|}{0.5} & \multicolumn{2}{|c|}{1.0} & \multicolumn{2}{|c|}{2.0} & \multicolumn{2}{|c|}{4.0} \\
\hline & Recovery/\% & $\mathrm{RSD} / \%$ & Recovery/\% & $\mathrm{RSD} / \%$ & Recovery/\% & $\mathrm{RSD} / \%$ & Recovery/\% & $\mathrm{RSD} / \%$ \\
\hline DNSH & 86.2 & 4.7 & 93.5 & 7.9 & 87.6 & 8.8 & 99.0 & 6.3 \\
\hline SEM & 100.5 & 5.3 & 98.4 & 5.1 & 83.9 & 9.0 & 100.3 & 5.2 \\
\hline $\mathrm{AOZ}$ & 82.4 & 7.3 & 82.0 & 9.6 & 87.8 & 9.7 & 94.6 & 3.4 \\
\hline AHD & 95.0 & 5.0 & 100.2 & 6.5 & 93.6 & 7.7 & 97.8 & 8.0 \\
\hline $\mathrm{AMOZ}$ & 81.3 & 7.8 & 89.9 & 8.8 & 88.0 & 6.6 & 94.8 & 10.0 \\
\hline
\end{tabular}

\section{3 结论}

硝呋索尔为新发现的呋喃类药物, 我国目前尚 未建立对该药物的研究方法。本方法采用液相色 谱-三重四极杆串联质谱法对水产品中的包括硝呋 索尔在内的 5 种硝基呋喃类药物的代谢物同时进行 定性和定量分析,对保障我国食品安全具有重要意 义。该方法前处理简单、重复性好、检出限低,可实 现同时检测水产品中 5 种硝基呋喃类药物的代谢物 残留量。使用 $\mathrm{AMOZ} 、 \mathrm{AHD} 、 \mathrm{SEM} 、 \mathrm{AOZ}$ 的同位素内 标, 减小了前处理过程的影响,使定量更加准确。选 用 SAH 作为 DNSH 的内标, 该物质由硝基呋喃类药 物硝呋奇特经过水解获得, 可得到满意的分析结果。

\section{参考文献:}

[1] Dai X, Li G J. Jilin Water Resources (戴欣, 李改娟. 吉林水 利), 2011(9): 61

2] Jiang Y, Ding T, Xu J Z, et al. Animal Husbandry \& Veterinary Medicine (蒋原, 丁涛, 徐锦忠, 等. 畜牧与兽医) , 2008, 40(2): 34

[3 ] Zuidema T, Mulder P P J, van Rhijn J A, et al. Anal Chim Acta, 2005, 529: 339

4] Aila O, Shitandi A, Mahungu M S, et al. Food Control, $2009,20: 543$

[5] Zhang L, Li J, Wang X, et al. Shanghai Journal of Animal Husbandry and Veterinary Medicine (张玲, 李劼, 王崟金, 等. 上海畜牧兽医通讯)，2008(5): 44

[6] Wang Y, Cai Y Q, Jia D F, et al. Analysis Laboratory (王 媛, 蔡友琼, 贾东芬, 等. 分析试验室), 2009, 28(12):86
[7] McCracken R J, Kennedy D G. J Chromatogr A, 1997, 771 : 349

[8] Barbosa J, Moura S, Barbosa R, et al. Anal Chim Acta, $2007,586: 359$

[9] Zhang H C, Li J, Yan X D, et al. Chinese Journal of Veterinary Medicine (张会彩, 李军, 间晓东, 等. 中国兽医杂志), $2011,47(5): 72$

[10 ] Xia X, Li X, Zhang S, et al. J Chromatogr A, 2008, 1208: 101

[11] Mottier P, Khong S, Gremaud E, et al. J Chromatogr A, $2005,1067: 85$

[12 ] Lin L M, Lin H C, Liu X T, et al. Chinese Journal Analytical Chemistry (林黎明，林回春，刘心同，等. 分析化学), $2005,33(5): 707$

[13 ] Cooper K M, Samsonova J V, Plumpton L, et al. Anal Chim Acta, 2007, 592: 64

[14] Liu A C, Liu C, Sang L Y, et al. Modern Agricultural Science and Technology (柳爱春，刘超，桑丽雅，等. 现代农 业科技)，2012(23): 265

[15] Zhao Z M, Luo X Q, Wang S L, et al. Shanghai Journal of Animal Husbandry and Veterinary Medicine (赵正苗, 罗晓 琴，汪善良，等.上海畜牧兽医通讯)，2012(5): 4

[16] Verdon E, Couedor P, Sanders P. Anal Chim Acta, 2007, $586: 336$

[17] Kaufmann A, Butcher P, Maden K, et al. Mitteilungen aus Lebensmitteluntersuchung und Hygiene, 2004, 95: 135

[18 ] Peng T, Qiu Y M, Li S J, et al. Inspection and Quarantine Science (彭涛，邱月明，李淑娟，等。检验检疫科学), 2003, 13(6) : 24

[19] Radovnikovic R, Moloney M, Byrne P, et al. J Chromatogr B, 2011, 879: 159

[20] Rodziewicz L. J Chromatogr B, 2008, 864: 156

[21 ] Xing L H, Sun W H, Li Z X. Environmental Chemistry (邢 丽红, 孙卫红, 李早新. 环境化学), 2011, 30(6) : 1202 